\title{
Interactions between endogenous and exogenous attention during vigilance
}

\author{
Katherine A. Maclean and Stephen R. Aichele \\ University of California, Davis, California \\ DAVID A. BRIDWELL \\ University of California, Irvine, California
}

AND

George R. Mangun, Ewa WojCiUlik, AND Clifford D. SARon
University of California, Davis, California

The ability to remain vigilant over long periods of time is critical for many everyday tasks, but controlled studies of visual sustained attention show that performance declines over time when observers are required to respond to rare stimulus events (targets) occurring in a sequence of standard stimulus events (nontargets). When target discrimination is perceptually difficult, this vigilance decrement manifests as a decline in perceptual sensitivity. We examined whether sudden-onset stimuli could act as exogenous attentional cues to improve sensitivity during a traditional sustained attention task. Sudden-onset cues presented immediately before each stimulus attenuated the sensitivity decrement, but only when stimulus timing (the interstimulus interval [ISI]) was constant. When stimulus timing was variable, exogenous cues increased overall sensitivity but did not prevent performance decline. Finally, independent of the effects of sudden onsets, a constant ISI improved vigilance performance. Our results demonstrate that exogenous attention enhances perceptual sensitivity during vigilance performance, but that this effect is dependent on observers' being able to predict the timing of stimulus events. Such a result indicates a strong interaction between endogenous and exogenous attention during vigilance. We relate our findings to a resource model of vigilance, as well as to theories of endogenous and exogenous attention over short time periods.

The ability to focus attention is a central feature of human cognition. Attention is multifaceted, sometimes driven by internal goals and sometimes by external stimulation. The time course of attention also varies from periods of time that are quite short (a few milliseconds) to longer periods (a few seconds or minutes). Goal-driven attention is referred to as top-down or endogenous attention, whereas stimulus-driven attention is referred to as bottom-up or exogenous attention, driven by external events in the environment (e.g., Posner \& Cohen, 1984). Allocating attention over short time periods can be referred to as phasic orienting (Posner, 1980), whereas maintaining attention over longer time periods is referred to as sustained attention, or vigilance (Parasuraman, 1986). The interactions among these varieties of attention come into play in our momentary experience as our goals and intentions compete with the attractions of the environment over milliseconds, seconds, and minutes (for a review, see Ruz \& Lupiáñez, 2002). The primary focus of the present study was to investigate the effects of endogenous and exogenous attention during vigilance, which we deem critical to a comprehensive understanding of short- and long-duration attention.
Endogenous attention can be engaged explicitly to select one thing over another (e.g., attend to one location and ignore others; Posner, 1980), as well as to follow a particular instructional set or general rule (e.g., attend to and report the color of the presented word; Stroop, 1935). Endogenous attention can also be guided by spatiotemporal regularities that are learned implicitly over time (Large \& Jones, 1999). In contrast to both explicit and implicit forms of endogenous attention, exogenous attention refers to effects that are generated externally by the physical properties of stimuli (e.g., brightness, color, shape). Exogenous attention allows novel or salient information (e.g., a sudden change in luminance; Jonides \& Yantis, 1988) to transiently interrupt goal-directed behavior. The dynamic and flexible interplay between endogenous and exogenous attention has been well studied in relation to visuospatial selective attention over short time periods (Folk, Leber, \& Egeth, 2002; Folk, Remington, \& Johnston, 1992), and this interaction continues to be characterized in electrophysiological and brain imaging studies (e.g., Hopfinger \& West, 2006; Serences et al., 2005) that seek to functionally delineate the brain networks supporting these attention systems. Current theories posit that endogenous and exogenous attention systems inter-

K. A. MacLean, katherine.a.maclean@gmail.com 
act and compete over short time periods to guide behavior (Corbetta, Patel, \& Shulman, 2008; Corbetta \& Shulman, 2002), with the result of that interaction being real-time prioritization of attentional focus and stimulus processing based on both endogenous and exogenous factors (Fecteau \& Munoz, 2006; Gottlieb, 2007).

However, less is known about how exogenous and endogenous attention systems interact over longer periods of time. The ability to maintain high levels of focused attention or vigilance over long periods of time underlies success on a range of tasks, from reading to airport security monitoring, but concentration often fails in such situations (e.g., Mackworth, 1948). Moreover, sustained attention is deemed to be effortful and stressful when one is required to maintain high levels of performance (Grier et al., 2003; Szalma et al., 2004; Warm, Parasuraman, \& Matthews, 2008). Among the major theories of vigilance, the resource model (Davies \& Parasuraman, 1982) proposes that the drop-off in performance over time - the vigilance decrement - is a result of the exhaustion of information-processing resources that are not replenished over time. Although the exact nature of these attentional resources is still unclear, recent evidence from brain imaging studies supports the idea that resources diminish over time (e.g., reductions in cerebral blood flow related to the vigilance decrement; Hitchcock et al., 2003). Assuming that endogenous resources are limited and that vigilance requires attentional effort, it is important to determine whether endogenous and/or exogenous attention manipulations might improve vigilance performance.

Robertson, Mattingley, Rorden, and Driver (1998) examined the relationship between vigilance and spatial attention and demonstrated that improvements in sustained attention can lead to better spatial attention (e.g., less severe symptoms in patients with hemispatial neglect after sustained attention training). But the reverse - that spatial attention benefits may lead to better vigilance performance-has received less consideration. Exogenous and endogenous cues may improve vigilance in different ways. For example, the deleterious effects of insufficient resources over time during a vigilance task may be offset by manipulating exogenous attention, such as by inserting attentiongrabbing, salient stimuli to transiently prime resources. In this vein, previous research on the effects of exogenous stimulation during vigilance has shown promising effects for procedures that capitalize on the effects of strong alerting cues (e.g., a loud sound linked to paying more attention; O’Connell et al., 2008; Robertson, Tegnér, Tham, Lo, \& Nimmo-Smith, 1995). However, no researchers have looked at the effects on vigilance of simple suddenonset visual cues like those used in classic studies of exogenous attention benefits (e.g., Posner \& Cohen, 1984). Although exogenous cues may transiently prime resources, endogenous cues may help the observer conserve attentional resources over time. Previous studies using various forms of endogenous cuing, such as the presentation of explicit warning cues (Hitchcock, Dember, Warm, Moroney, \& See, 1999; Hitchcock et al., 2003) or implicit manipulations of temporal predictability (Helton et al., 2005; Scerbo, Warm, Doettling, Parasuraman, \& Fisk, 1987) have yielded similar results: Endogenous attention cues reduce the vigilance decrement. Despite this empirical evidence, the interaction between exogenous attention and endogenous attention during vigilance remains unclear, primarily owing to the fact that no investigators have examined effects of exogenous and endogenous cuing in the same study. Thus, this was the purpose of the present study. We directly investigated the interaction between endogenous and exogenous attention during vigilance in order to better understand how manipulations of both forms of attention would affect the classic performance decrement. Specifically, we investigated (1) whether simple sudden-onset visual events could improve sustained attention performance (exogenous attention manipulation) and (2) whether benefits due to sudden onsets would interact with fluctuations in attention as a function of the predictability of stimulus timing (endogenous attention manipulation).

\section{Theories of Sustained Attention}

Most visual sustained attention tasks are variations of the original Mackworth clock test (Mackworth, 1948), used to test vigilance performance in radar operators. In the clock test, observers monitored a pointer moving in single step increments across a blank clock face for periods of up to $2 \mathrm{~h}$ and were instructed to indicate when they detected a rare double jump in the movement of the pointer. Current sustained attention tasks share several core design elements of the clock test, in that they require participants to monitor the sequential display of neutral, nontarget stimulus events over a long period of time and initiate responses to rare target events occurring unpredictably. Typically, performance declines over time, with the greatest decrement in correct detections occurring within the first 15-20 min (Davies \& Tune, 1969). Of particular interest are those tasks that produce a decline in perceptual sensitivity $\left(d^{\prime}\right.$ or $\left.A^{\prime}\right)$, indicating that participants are actually detecting fewer targets over time, not simply adopting a more conservative response criterion (Green \& Swets, 1966).

Since Mackworth's (1948) initial investigation, a multitude of experiments have contributed to a better understanding of why humans are not very good at maintaining voluntary attention for long periods of time. Several theoretical models have been proposed to explain the classic vigilance decrement, including the arousal model (Frankmann \& Adams, 1962), in which the decrement is viewed as a consequence of reductions in general alertness over time, and the mindlessness model (Manly, Robertson, Galloway, \& Hawkins, 1999; Robertson, Manly, Andrade, Baddeley, \& Yiend, 1997), in which the decrement is viewed as the result of failures of a supervisory attention system to appropriately direct awareness toward a relatively boring, undemanding task. Experimental findings seem to challenge both of these theories and instead support an attentional resource model (Davies \& Parasuraman, 1982) as the best explanation of performance decrements. In recent studies, questionnaire and self-report measures have been used in combination with vigilance tasks to test key predictions of these competing theories to more fully characterize sustained attention as resource demanding and effortful (Warm et al., 2008). For example, adding irrelevant stimuli to reduce task monotony (i.e., increase overall arousal) 
does not improve vigilance performance (Smit, Eling, \& Coenen, 2004), in contradiction to the arousal theory of vigilance. With respect to the mindlessness theory, studies have shown that observers find vigilance tasks to be effortful rather than undemanding (Grier et al., 2003; Szalma et al., 2004), that performance is influenced by implicit stimulus patterns during the task (i.e., observers do not withdraw awareness from the task; Helton et al., 2005), and that self-reported mind wandering (e.g., task-unrelated thought) is not related to the vigilance decrement (Helton \& Warm, 2008). In addition, brain imaging studies offer direct evidence for the model's assumption of a reduction in attentional resources. Although it was suggested in early studies that blood flow reduces over time during a task (Paus et al., 1997), Hitchcock et al. (2003) more recently showed that reductions in cerebral blood flow were directly related to the extent of the vigilance decrement and that the change in blood flow was specific to task factors that had previously been interpreted to increase resource demands (e.g., difficult target discrimination). In line with the resource model, functional imaging studies of sustained attention over shorter periods of time (e.g., several minutes) point to specific reductions in activity in attentional control regions linked to subsequent lapses of attention (Weissman, Roberts, Visscher, \& Woldorff, 2006).

\section{Factors Affecting Sustained Performance}

A combination of experimental (e.g., Parasuraman, 1979) and meta-analytic approaches (e.g., See, Howe, Warm, \& Dember, 1995) have worked to identify the key factors that increase resource demands and thus promote declines in perceptual sensitivity during vigilance. Event rate (i.e., the rate of stimulus events that must be inspected for the presence of the rare target event) has been judged to be one of the prepotent parameters that affect vigilance performance, with high event rate ( $>24$ events per minute; Parasuraman \& Davies, 1977) increasing the resource demands of the task and thereby increasing the perceptual sensitivity decrement (Davies \& Parasuraman, 1982; Parasuraman \& Davies, 1977; See et al., 1995; Warm \& Jerison, 1984). Perceptual sensitivity also declines more reliably in tasks in which the target discrimination process loads memory (Parasuraman, 1979). For example, when the currently visible stimulus in a series of successive presentations is compared with a standard nontarget held in memory (e.g., detecting a short line target occurring in a sequence of long line nontargets), observers perform more poorly over time than they do in those tasks that require a comparative judgment based only on the currently visible stimuli (e.g., detecting a line length difference between two lines presented at the same time). Parasuraman (1979) described these tasks as successiveand simultaneous-type presentation, respectively. Importantly, decrements may be observed in simultaneous-type tasks if target discrimination is difficult. Parasuraman and Mouloua (1987) demonstrated that targets that are initially perceptually difficult to discriminate from nontargets become more difficult to detect over time than targets that are easy to discriminate, regardless of successive- versus simultaneous-type presentation. Even tasks with numeric targets - which do not consistently produce decrements in healthy participants because these targets are easy to discriminate - promote declines in perceptual sensitivity if the targets are highly degraded and thus rendered more difficult to discriminate from nontarget numbers (Nuechterlein, Parasuraman, \& Jiang, 1983). In this way, sensitivity over time is generally susceptible to the resource demand imposed by target discrimination.

\section{Perceptual Benefits of Endogenous Spatial Attention}

In many studies outside of the vigilance domain, the extent to which endogenous attention improves performance under conditions of low sensitivity (e.g., low contrast, short stimulus duration) has been investigated. In the present study, we interpret internally represented forms of attention, either explicit (voluntary) or implicit, as aspects of endogenous attention, although we will discuss the behavioral and neural effects of each in turn. In designs that explicitly manipulate attention on a trial-by-trial basis, voluntary spatial attention improves the accuracy and speed with which stimuli are detected (e.g., Posner, 1980). Voluntary attention can also be deployed to varying degrees, with associated perceptual sensitivity benefits commensurate with the amount of attention (e.g., 100\% vs. $75 \%$ ) to a given spatial location (Mangun \& Hillyard, 1990). Event-related potential (ERP) studies in humans have shown that voluntary attention improves sensitivity by enhancing the gain of attended as compared with unattended stimulus representations at early levels of visual processing. For example, many studies have found amplitude enhancements of early latency visual evoked responses to stimuli that occur in the location of voluntary attention (Mangun \& Hillyard, 1991; Martínez, Di Russo, Anllo-Vento, \& Hillyard, 2001; Van Voorhis \& Hillyard, 1977). Moreover, behavioral and neurophysiological enhancements occur at the fovea with focused rather than distributed attention (Miniussi, Rao, \& Nobre, 2002), demonstrating that directed voluntary attention can enhance processing even when spatial resolution is already high. In light of the benefits of endogenous attention, it is perhaps not surprising that performance on sustained attention tasks suffers, since voluntary attention wanes over time. Indeed, an overall lack of focused, voluntary attention can lead to severe deficits in perceptual processing of fully visible and distinctive objects, a robust phenomenon that has been referred to as inattentional blindness (Most et al., 2001; Simons \& Chabris, 1999).

Endogenous attention can also be guided by regularities or patterns that are implicitly learned over time, such as the probability of a target item appearing in a particular spatial location (Chun \& Jiang, 1998; Geng \& Behrmann, 2005; Hoffmann \& Kunde, 1999) or temporal patterns that predict when task-relevant stimuli will occur (Martin et al., 2005; Olson \& Chun, 2001). Like explicit manipulations of attention, implicit manipulations of endogenous attention have been shown to be strong predictors of behavioral improvements. For example, Geng and Behrmann showed spatial probability to robustly influence attention, with behavioral improvements (shorter reaction times [RTs], better accuracy) occurring at likely rather than unlikely loca- 
tions. Moreover, they showed that implicit cuing through spatial probability had just as strong an influence on attention as did explicit endogenous cues and was effective in reducing distraction during task performance.

\section{Perceptual Benefits of \\ Exogenous Spatial Attention}

Exogenous selective attention also improves performance under conditions of low sensitivity. Behaviorally, exogenous attention improves detection of, and facilitates responses to, salient sudden-onset stimuli (Jonides \& Yantis, 1988; Posner \& Cohen, 1984). In addition, suddenonset cues that occur within $\sim 200 \mathrm{msec}$ of an upcoming stimulus have been shown to enhance apparent contrast (Carrasco, Ling, \& Read, 2004; Carrasco, Penpeci-Talgar, $\&$ Eckstein, 2000; Ling \& Carrasco, 2006) and to increase spatial resolution (Carrasco, Williams, \& Yeshurun, 2002; Yeshurun \& Carrasco, 1999), thus improving perceptual sensitivity to stimuli that occur in the location of the cue. Although most studies of exogenous attention have examined peripheral cuing, sudden onsets have been shown to capture attention at the fovea as well. For example, Coull, Frith, Büchel, and Nobre (2000) demonstrated that targets that occurred unexpectedly at fixation while participants prepared to attend to a later point in time (but still at fixation) captured attention in a stimulus-driven manner that was similar to exogenous capture in the periphery. Visual cortex activity to the unexpected targets (as measured using fMRI) was preferentially enhanced, supporting the idea that exogenous attention results in sensitivity and gain enhancements in visual processing, even at the fovea.

\section{Interactions Between Endogenous and Exogenous Attention}

In cases of highly focused voluntary attention, observers are usually able to resist distraction from salient, abrupt onsets outside the focus of attention (Theeuwes, 1991; Yantis \& Jonides, 1990). However, several lines of research indicate that endogenous and exogenous visuospatial attention interact, in that the consequences of sudden-onset salient events (exogenous attention) can be modulated by the current goal state (endogenous attention). Although these combined influences may sometimes lead to performance impairments, such as when one is distracted from paying attention to a certain location in space by a salient interruption in the periphery (van der Lubbe \& Postma, 2005), exogenous orienting is not truly automatic (for a review, see Santangelo \& Spence, 2008). Instead, the total impact of a sudden-onset event on endogenous attention has been shown to depend on how much the event shares in common with the target of voluntary attention (e.g., the target and the sudden-onset distractor are the same color), a mechanism labeled contingent attentional capture (Folk et al., 2002). Relevant to our present investigation, such sudden onsets can lead to performance enhancements, such as when endogenous and exogenous attention cues converge on the same location. In a study of directed spatial attention, Hopfinger and West (2006) showed that brief exogenous cues that occurred in the location of voluntary attention (e.g., left or right visual field) amplified the behavioral benefits of endogenous attention: Participants were faster to respond to an upcoming target when an exogenous cue occurred in the location where they were already paying voluntary attention. Overall, these findings demonstrate that endogenous and exogenous attention systems together optimize both behavior and the distribution of neural resources.

\section{Sustained Interactions Between \\ Endogenous and Exogenous Attention}

Although endogenous and exogenous attention manipulations have been shown to impact sustained performance when examined separately, it remains to be determined how these systems interact over long periods of time when endogenous attention resources are limited. Manipulations of implicit endogenous attention appear to be strong predictors of better sustained attention. Namely, observers benefit from temporal regularities in the structure of stimulus presentation, showing better performance over time when stimuli occur at a constant, rhythmic rate (Scerbo et al., 1987) or when targets occur at constant intervals (e.g., one target every $30 \mathrm{sec}$; Helton et al., 2005). In terms of manipulations of exogenous attention, some research has focused on the negative impact of unexpected, exogenous distractors on vigilance performance (Davies \& Tune, 1969; Warm \& Jerison, 1984). Evidence also exists in support of the opposite effects: Transient enhancements in attention resulting from salient cues can improve sustained performance in both normal populations (O'Connell et al., 2008) and in patients with right hemisphere brain damage (Robertson et al., 1995). However, in these studies, the benefits to sustained performance were the result of intensive training with loud auditory cues that primarily affected overall physiological arousal (i.e., increases in skin conductance response; O'Connell et al., 2008). Whether the kind of simple sudden-onset visual events such as those used in cuing studies of exogenous attention over short time periods can change the trajectory of sustained attention performance and how these exogenous attention benefits might interact with fluctuations in endogenous attention are therefore still open questions.

\section{The Present Study}

The overarching goal of the present study was to examine the independent and combined effects of manipulations of exogenous and endogenous attention during vigilance. Our first aim was to demonstrate how exogenous attention affects performance on a sustained attention task requiring a demanding perceptual discrimination. In Experiment 1 , we compared performances on two versions of a sustained attention task that differed in display features designed to capture exogenous attention. We hypothesized that sudden onsets would improve sensitivity by drawing attention exogenously to the task and would thus attenuate the usual vigilance decrement that occurs when observers fail to maintain high levels of voluntary attention. Our second aim was to determine how exogenous and endogenous attention manipulations interact to change vigilance performance. In Experiment 2, we varied interstimulus timing (ISI) to determine whether the benefit due to sud- 
den onsets depended on precise and predictable temporal information about when to allocate endogenous attention. To anticipate our results, we found that exogenous cues improved perceptual sensitivity over time when ISI was constant (Experiment 1) but that a variable ISI reduced this benefit (Experiment 2). Finally, in a control experiment (Experiment 3), we varied the luminance of the exogenous cue to confirm that improvements in perceptual sensitivity were due to attentional capture, rather than to sensory activation.

\section{EXPERIMENT 1}

The purpose of this experiment was to examine the effect of simple sudden-onset exogenous attention cues on a perceptually demanding sustained attention task. One of the necessary objectives was to create target stimuli that were equally demanding across individuals. Vigilance researchers have often noted striking individual differences in baseline performance (Davies \& Tune, 1969; Parasuraman, 1986), which poses serious problems for comparing performances over time across individuals. Simply put, a target that is initially difficult for one person to discriminate might be easy for another person to discriminate and these two people will experience very different resource demands in maintaining endogenous attention for a long period of time. Although some studies have set target parameters based on average group discrimination performance (Parasuraman \& Mouloua, 1987), vigilance tasks do not commonly use individually determined target parameters. The few exceptions have employed adaptive threshold procedures to change a target stimulus parameter (e.g., brightness) throughout the task, in effect making the targets easier to detect in order to compensate for performance decline (Bakan, 1955; Berger \& Mahneke, 1954; Frome, MacLeod, Buck, \& Williams, 1981; Wiener, 1973). In contrast, in our approach here, we determined target parameters for each participant using an individual discriminability threshold procedure and then presented that threshold-level target throughout the duration of the subsequent sustained attention task. This novel design ensures equivalent attentional and perceptual demands across individuals, which makes the task highly sensitive to fluctuations of attention over time. In this first experiment, we implemented threshold-based targets and compared perceptual sensitivity over time on two versions of a sustained attention task that differed in display features that were designed to capture exogenous attention. We predicted that perceptual sensitivity would decline less in the sustained attention task, in which brief sudden-onset cues were displayed immediately before a potential target stimulus because of the influence of exogenous attention.

\section{Method}

\section{Participants}

Seventeen volunteers (mean age $=19.5$ years, range $=18-32$ years, 15 female) from the University of California, Davis, gave informed consent and participated in exchange for course credit. All of the participants had normal or corrected-to-normal vision and were free from neurological insult. Analyses of performance over time did not include participants who could not initially perform the task to criterion. One participant (female) performed below the initial accuracy cutoff (at least $50 \%$ correct detections) during the first $4 \mathrm{~min}$ of one version of the sustained attention task and was excluded from all analyses. Data from the remaining 16 participants are presented here.

\section{Stimuli}

For all tasks, participants were seated comfortably in a darkened sound-attenuating booth, with a chinrest ensuring a viewing distance of $57 \mathrm{~cm}$. Presentation software (Neurobehavioral Systems, www .neurobs.com) was used to control the display of all stimuli on a 53-cm CRT monitor (Eizo FlexScan F980) at a refresh rate of $60 \mathrm{~Hz}$. Responses were made using the top right key on a ProPad gamepad response device. Participants $(N=16)$ completed two versions of a successive-presentation (Parasuraman, 1979) sustained attention task that required buttonpresses to rare targets (uninterrupted task duration for each version $=32 \mathrm{~min}$ ). During the task, single lines (light gray, $1.31 \mathrm{~cd} / \mathrm{m}^{2}$ ) were presented at the center of the screen against a black background $\left(0.02 \mathrm{~cd} / \mathrm{m}^{2}\right)$ while participants fixated a small red square (see Figure 1). The fixation square was continuously visible at the center of the screen, and participants were instructed to maintain their gaze on the square throughout the task. Stimuli were presented at a high event rate $(30$ events $/ \mathrm{min}$; stimulus duration $=150 \mathrm{msec}$, interstimulus interval [ISI] $=1,850 \mathrm{msec}$ ), which is considered to be resource demanding and has been previously shown to promote de-

\section{Target trial}
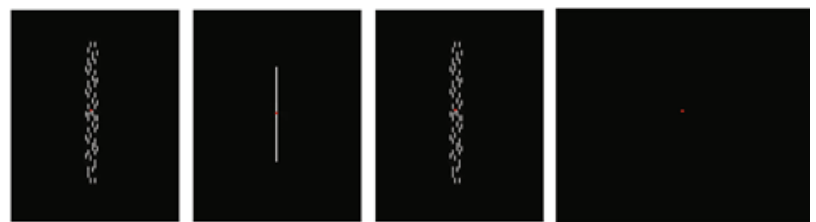

Mask

$100 \mathrm{msec}$
Short line

$150 \mathrm{msec}$
Mask

$100 \mathrm{msec}$
Fixation dot

$1,650 \mathrm{msec}$

$|S|=1,850 \mathrm{msec}$

Figure 1. Stimuli and timing for the transient display version of the sustained attention task. Sequence of events is shown for one nontarget trial (long line stimulus) and one target trial (short line stimulus). The transient mask appeared before and after every line (both targets and nontargets) as depicted. In the stable display version, the mask was presented continuously throughout the entire 1,850-msec interstimulus interval (ISI; e.g., in place of the sole fixation square shown in the figure). Stimuli are shown in white for illustrative clarity, but were presented in light gray during the tasks; the fixation square was presented in red in Experiment 1 and yellow in Experiment 2. In Experiment 2, the ISI varied randomly across trials $(M=1,850 \mathrm{msec}$, range $=1,350-2,350 \mathrm{msec})$. The fixation square was continuously visible at the center of the screen during all tasks. 
clines in perceptual sensitivity over time (Parasuraman, 1979). Long nontarget lines $\left(4.5^{\circ}\right)$ were presented $90 \%$ of the time, and short target lines $\left(M=3.4^{\circ}\right.$, range $\left.=3.2^{\circ}-4.1^{\circ}\right)$ were presented $10 \%$ of the time. Line width $\left(0.035^{\circ}\right)$ did not differ between short and long lines. Two target lines occurred pseudorandomly every 20 trials, with the restriction that only two short lines could occur consecutively at any point in the task. The instructions emphasized accuracy in making simple button responses with the right index finger to the target short lines. Each participant performed two versions (order counterbalanced) of the sustained attention task that differed according to display features: The stable version displayed a mask pattern (same brightness as the line stimuli) during the entire ISI, and the transient version displayed the same mask pattern for $100 \mathrm{msec}$ before and after each line stimulus, with a blank intervening screen (see Figure 1). The sudden onset of the mask before the line in the transient display was designed as an exogenous attention cue, in line with previous findings showing a sudden change in luminance contrast to be one of the strongest forms of exogenous attentional cuing (Steinman, Steinman, \& Lehmkuhle, 1997). The presentation of the mask following the line was included to equate visibility between the transient and stable displays (i.e., to prevent a persisting afterimage in the transient display). The mask was composed of many short lines positioned throughout a $5.0^{\circ} \times 1.0^{\circ}$ space; each short line was $0.07^{\circ}$ wide and ranged in height from $0.28^{\circ}$ to $0.45^{\circ}$. The mask pattern was never present during line stimulus presentation. The Michelson contrast ratio between the line/mask stimuli and the black background was 97\% (Coren, Ward, \& Enns, 1999).

\section{Procedure}

Threshold procedure. The length of the short target line was set to each participant's discrimination threshold using a self-paced 3-down:1-up staircase procedure that determined an $80 \%$ accuracy threshold (see Leek, 2001). Display characteristics and event durations were matched to the stable and transient versions of the sustained attention task. During each trial of the threshold task, a warning signal (enlargement of the red fixation square) preceded two sequential lines at fixation, and participants judged the length difference between the two lines, indicating whether the length was the same or different with the top left (left index finger) and top right (right index finger) gamepad buttons, respectively. Each trial was composed of (1) a long and a short line (order randomized), (2) two short lines, or (3) two long lines. Participants made an untimed response and then received sound feedback (a ding sound for correct, a whoosh sound for incorrect) indicating response accuracy. The length of the short line varied throughout the task in $\sim 0.07^{\circ}$ steps (the first short line subtended $3.6^{\circ}$, possible range $=3.2^{\circ}-4.4^{\circ}$ ), with increments in line length occurring after three successive correct responses and decrements in line length occurring after each incorrect response. The task terminated after eight length reversals (i.e., a switch from an increment in line length to a decrement in line length). The length of the long line was the same as that in the main task and never varied.

Testing procedures. Participants were introduced to the task through brief verbal instructions and practice of the threshold procedure (20 trials with the short line length held constant at a length that would be easily detectable by all participants). Participants then completed the threshold procedure $(\sim 10 \mathrm{~min})$ followed by practice on the sustained attention task with the target line length set to their threshold $(5 \mathrm{~min})$. An extra $5 \mathrm{~min}$ of practice was allowed if participants correctly detected fewer than $75 \%$ of the targets. ${ }^{1}$ Participants then performed the first version (e.g., stable display) of the sustained attention task for 32 min (960 trials) without sound feedback or breaks. This sequence of tasks was repeated for the second version (e.g., transient display) of the sustained attention task after a $15-\mathrm{min}$ break. Order of display version (stable or transient first) was counterbalanced across participants.

\section{Data Analysis}

Hit and false alarm rates were calculated over four contiguous blocks (240 trials each) for each version (transient vs. stable) of the sustained attention task. Hits were defined as correct detec- tions of targets (buttonpresses to the short lines), and false alarms were defined as incorrect buttonpresses to nontarget long lines. The nonparametric index of perceptual sensitivity, referred to as $A^{\prime}$, was calculated from the hit and false alarm rates (Stanislaw \& Todorov, 1999) and served as the main dependent measure of performance over time. The nonparametric index of response bias, $\beta_{\mathrm{D}}^{\prime \prime}$ (See, Warm, Dember, \& Howe, 1997), was also calculated and used to assess changes in response tendencies over time. Finally, correct RTs to targets were examined; RTs were trimmed to remove the top and bottom $5 \%$ of outliers on a cell-by-cell basis for each individual. A multivariate ANOVA (Maxwell \& Delaney, 2004, pp. 671-675, 682-751), which implies no assumption about compound sphericity, was used to assess all within- and between-subjects effects, and Bonferroni correction was used to control the familywise error rate for all post hoc analyses (e.g., $\alpha=.05 / 2$ when testing two simple main effects to follow up a significant interaction).

\section{Results}

\section{Threshold}

The threshold procedure successfully produced a $\sim .80$ hit rate in $87 \%$ of the participants ${ }^{2}$ during the first block (240 trials) of the corresponding sustained attention task $(M=.77, S D=.14)$. Difference thresholds - the visual angle difference between the standard long line and the participant's threshold-level short line-were subjected to a mixed-model ANOVA with the within-subjects factor of display version (stable vs. transient) and the betweensubjects factor of order of display version (stable first vs. transient first). The only significant source of variance was an interaction between display and order $[F(1,14)=$ $18.29, p<.001]$, which revealed that thresholds were lower (i.e., there was a smaller difference in visual angle between the two lines) on the threshold task that was performed second regardless of display version (Table 1). The results suggest that participants' perceptual thresholds improved with exposure to the tasks in general, but did not differ according to display version.

\section{Order Effects on Performance}

All measures of performance over time were subjected to a mixed-model ANOVA with the within-subjects factors of time (four blocks) and display version and the between-subjects factor of order of display version. It was first important to determine whether task order affected performance. In terms of accuracy $\left(A^{\prime}\right)$, the omnibus ANOVA showed no main effects of order but did show a significant interaction between order and display version $[F(1,14)=6.57, p=.022]$. Overall accuracy was higher in the task that was performed first, regardless of display version (see Table 1), although order did not modulate

Table 1

Order Effects on Threshold and Performance

\begin{tabular}{|c|c|c|c|c|}
\hline \multirow[b]{2}{*}{ Task Order } & \multicolumn{2}{|c|}{$\begin{array}{l}\text { Difference } \\
\text { Threshold }^{\mathrm{a}}\end{array}$} & \multicolumn{2}{|c|}{$\begin{array}{c}\text { Overall Perceptual } \\
\text { Sensitivity }\left(A^{\prime}\right)\end{array}$} \\
\hline & $\begin{array}{l}\text { First } \\
\text { Task }\end{array}$ & $\begin{array}{c}\text { Second } \\
\text { Task }\end{array}$ & $\begin{array}{l}\text { First } \\
\text { Task }\end{array}$ & $\begin{array}{c}\text { Second } \\
\text { Task }\end{array}$ \\
\hline Stable first & 1.09 & 0.88 & .943 & .917 \\
\hline Transient first & 1.06 & 0.79 & .936 & .890 \\
\hline
\end{tabular}

aVisual angle difference between the long line and the $80 \%$-thresholdlevel short line obtained through a 3-down:1-up staircase procedure. 
performance over time. Because of this significant effect, order was retained as a between-subjects factor in all subsequent analyses of accuracy. Order did not influence RTs (all $p \mathrm{~s}>.08$ ) and was excluded from RT analyses. ${ }^{3}$

We implemented the threshold approach to equate task difficulty, so there should be no consistent or predictable relationship between threshold and subsequent accuracy (i.e., different threshold values should produce an equivalent level of accuracy across participants). We wanted to confirm that the decrease in overall accuracy observed for the second task was not related to the improvement in threshold. There were no significant correlations between threshold and overall accuracy either across all data points $(r=-.11, p=.54)$ or within the first $(r=-.037, p=$ $.89)$ or the second $(r=.22, p=.40)$ task separately. This result suggests that the changes in threshold and accuracy in the second task did not reflect a consistent relationship between threshold and overall performance across individuals (see the Discussion section).

\section{Performance Over Time}

Sustained attention performance was assessed by examining changes in perceptual sensitivity $\left(A^{\prime}\right)$ over time. Mean $A^{\prime}$ values during the initial block did not differ between display versions $(M=.940$ for transient and $M=$ .937 for stable, $t<1$ ), indicating that performance on the threshold task successfully matched initial performance. Perceptual sensitivity declined over time in both tasks, although the main effect of time in the omnibus ANOVA was a nonsignificant trend [Block 1, $M=.939$; Block 2, $M=$ .934 ; Block 3, $M=.917$; Block 4, $M=.907 ; F(3,12)=$ $3.13, p=.065]$. Importantly, the decline in $A^{\prime}$ over time was qualified by a significant interaction between time and display version $[F(3,12)=5.35, p=.015]$. Examination of the performance trajectories over time revealed that there was comparatively less performance decline in the transient version than in the stable version, in line with our prediction (see Figure 2). A test of the simple main effect of time within each display separately indicated that the performance decline was significant in the stable version $[F(3,12)=4.87$, $p=.019]$ but not in the transient version $[F(3,12)=1.27$, $p=.32]$. No other sources of variance were significant in the analysis of $A^{\prime}$. A similar model of response bias revealed nonsignificant effects of time $[F(3,12)=0.386, p=.76]$, display $[F(1,14)=1.02, p=.32]$, and a nonsignificant interaction between display and time $[F(3,12)=1.09, p=$ .38 ]. Thus, changes in response bias did not accompany the observed changes in perceptual sensitivity.

Although we encouraged participants to respond accurately rather than quickly, we wanted to confirm that differences in the change in perceptual sensitivity between the two displays could not be explained by changes in RT (a speed-accuracy trade-off). Mean correct RTs (correct detections of targets) were submitted to a repeated measures ANOVA with the within-subjects factors of time (Blocks 1-4) and display version. A main effect of time revealed that RTs significantly increased over time [Block 1, $M=691 \mathrm{msec}$; Block 2, $M=745 \mathrm{msec}$; Block 3, $M=748 \mathrm{msec}$; Block 4, $M=745 \mathrm{msec} ; F(3,13)=9.23$, $p=.002]$, but RTs did not differ according to display

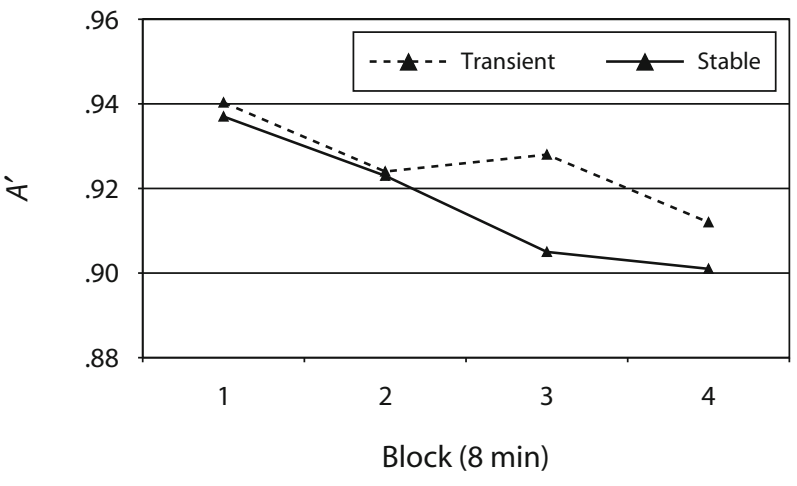

Figure 2. Changes in perceptual sensitivity $\left(A^{\prime}\right)$ as a function of time on task in Experiment 1. $A^{\prime}$ plotted over four contiguous 8-min blocks ( 240 trials each) for each display version (stable and transient).

$[F(1,15)=0.009, p=.92]$. In addition, the interaction between time and display version was not significant $[F(3,13)=2.37, p=.11]$. These results confirm that RTs did not become differentially faster in the stable display, which is the display version that showed the most decline in $A^{\prime}$ (i.e., there was no speed-accuracy trade-off). Because of the extremely low false alarm rates $(<5 \%)$, RTs for false alarms could not be reliably analyzed.

\section{Discussion}

The data show that threshold-based targets were an effective way to create sustained attention tasks that were perceptually challenging and maximally sensitive to individual changes in endogenous attention. We observed that the typical decline in performance over time was attenuated when display features of the transient task presumably facilitated exogenous capture of attention and thereby enhanced perception. When the positive effects of exogenous attention were removed in the stable display, the decline in perceptual sensitivity significantly increased. This finding is important, since it demonstrates for the first time that sustained discrimination depends not only on the availability of voluntary attention resources but, rather, is a result of an interaction between voluntary attentional control and the exogenous features of the task.

These data suggest that exogenous attention aided target discrimination by reducing demand on the endogenous attention system. Although it is clear that sudden onsets increased perceptual sensitivity, it is unclear how this sensitivity enhancement interacted with fluctuations in endogenous attention. Specifically, exogenous cues may have improved sensitivity to such an extent that the degree of endogenous attention on a given trial was less critical for correct target discrimination. However, the constant ISI between adjacent lines provided information about when to pay attention, and the impact of the exogenous cues on performance may have depended on this systematic modulation of endogenous attention. It is well known that temporal information can effectively guide the allocation of endogenous attention in a sustained performance context, such that performance over time is 
better when events occur in a rhythmic fashion (Scerbo et al., 1987). In effect, one can avoid effortful, continuous application of voluntary attention by using temporal information to take attention timeouts between stimulus presentations in order to preserve attentional resources over time. In addition, the manipulation of endogenous attention by temporal regularity has been shown to occur independent of conscious awareness (Martin et al., 2005). Finally, some researchers have posited that the impact of a sudden-onset cue on attentional capture may be strongest when the observer is optimally prepared for the occurrence of the target (Milliken, Lupiáñez, Roberts, \& Stevanovski, 2003).

\section{EXPERIMENT 2}

The primary purpose of Experiment 2 was to examine the impact of predictable timing cues on the perceptual benefits due to sudden-onset cues observed in Experiment 1 . We introduced a variable ISI in Experiment 2 to prevent participants from taking precisely timed and efficient breaks in attention, thus increasing the overall demand on the endogenous attention system. This manipulation allowed us to assess the effect of sudden onsets on sustained performance, independent of predictable timing.

The secondary purpose of Experiment 2 was to clarify the nature of changes in threshold and overall accuracy observed in the second task in Experiment 1. Improvements in perceptual threshold on the second task suggested either short-term perceptual changes (i.e., true changes in threshold due to stimulus exposure) or practice effects (i.e., improved performance on the threshold procedure itself rather than true changes in threshold). In Experiment 2, we designed a more ecologically valid threshold procedure that would be less susceptible to procedure-specific practice effects. To test for any changes in threshold due to stimulus exposure, we also remeasured threshold immediately following completion of the sustained attention task. In Experiment 1, along with improvements in threshold, we observed lower overall accuracy in the second task. This pattern suggested a possible interaction between threshold changes and changes in accuracy. If improvements in threshold were mainly driven by procedure-specific practice effects, thresholds would be artificially lower on average, leading to a more difficult target during the sustained attention task and to lower overall accuracy. At the group level, this could be expressed as accuracy going down as thresholds get better, as we observed. (Note that we did not observe a consistent relationship between threshold and accuracy at the individual level as reflected in the nonsignificant correlation values reported in Experiment 1.) The decrease in accuracy could alternatively reflect fatigue; however, the 15 -min break between task versions should have restored performance to normal levels (e.g., Mackworth, 1948). Our between-subjects manipulation of display in Experiment 2 directly addressed practice effect and fatigue issues related to threshold and accuracy during sustained attention.

\section{Method}

\section{Participants}

Forty volunteers (mean age $=20$ years, range $=18-34$ years, 26 female) from the University of California, Davis, gave informed consent and participated in exchange for course credit. All participants had normal or corrected-to-normal vision and were free from neurological insult.

\section{Stimuli}

Task and stimulus parameters (including visual angle, contrast ratio, and average event rate) for these tasks were the same as those in Experiment 1, except for a few important modifications. First, a variable ISI was included, representing a range of $500 \mathrm{msec}$ above and below the interval used in Experiment 1 (range $=1,350$ 2,350 msec). Second, debriefing in Experiment 1 revealed that some participants attempted to assess line length by comparing the currently presented line with the background mask parts. To prevent this strategy, we vertically repositioned each of the lines forming the mask on every presentation. The change in position of each mask part $\left(0.07^{\circ} \times 0.28^{\circ}\right)$ was randomly chosen within a range of $-0.14^{\circ}$ to $+0.14^{\circ}$, with negative values indicating a downward shift and positive values indicating an upward shift. The subtle shift was not immediately noticeable but prevented the comparison strategy if a participant attempted it. Finally, the type of display (stable vs. transient) was manipulated between subjects ( $n=20$ in each task version) to avoid possible fatigue or order effects on performance. As in Experiment 1, the instructions emphasized accuracy in making simple responses with the left mouse button (right index finger) to the target short lines. Stimuli were presented on a 21 -in. CRT monitor (Viewsonic AccuSync 120) at a refresh rate of $60 \mathrm{~Hz}$. Line and mask stimuli were presented in light gray $\left(3.25 \mathrm{~cd} / \mathrm{m}^{2}\right)$ on a black background $\left(0.05 \mathrm{~cd} / \mathrm{m}^{2}\right.$; Michelson contrast ratio $\left.=97 \%\right)$.

\section{Procedure}

Threshold procedure. The length of the short target line was set to each participant's $80 \%$ discrimination threshold using a variant of Parameter Estimation through Sequential Testing (PEST; see Taylor \& Creelman, 1967). PEST is an adaptive thresholding procedure that dynamically adjusts the amount of change (step size) between testing levels on the basis of current task performance. This design ensures that testing levels quickly converge toward a threshold value. PEST is more efficient, accurate, and flexible than fixed thresholding methods such as the staircase procedure used in Experiment 1. In our application of PEST, the display characteristics, event durations, and response requirements of the threshold task exactly matched those of the corresponding sustained attention task, except that the target short line occurred more often ( $33 \%$ of the time). Participants received sound feedback indicating (1) correct detections (a ding sound), (2) target misses (a whoosh sound), and (3) incorrect responses to nontargets (a whoosh sound).

Testing procedures. Participants followed the same sequence of tasks as in Experiment 1 (i.e., threshold procedure and practice followed by the sustained attention task). Additionally, to assess shortterm changes in perceptual threshold, participants performed the threshold task again after completing the sustained attention task.

\section{Results}

\section{Threshold}

The PEST threshold procedure successfully produced a .80 hit rate in $95 \%$ of the participants ${ }^{4}$ during the first block of the corresponding sustained attention task $(M=$ $.81, S D=.13$ ). Difference thresholds were subjected to a mixed-model ANOVA with the between-subjects factor of display (stable vs. transient) and the within-subjects factor of exposure (before vs. after). No significant differences were found between thresholds tested before 


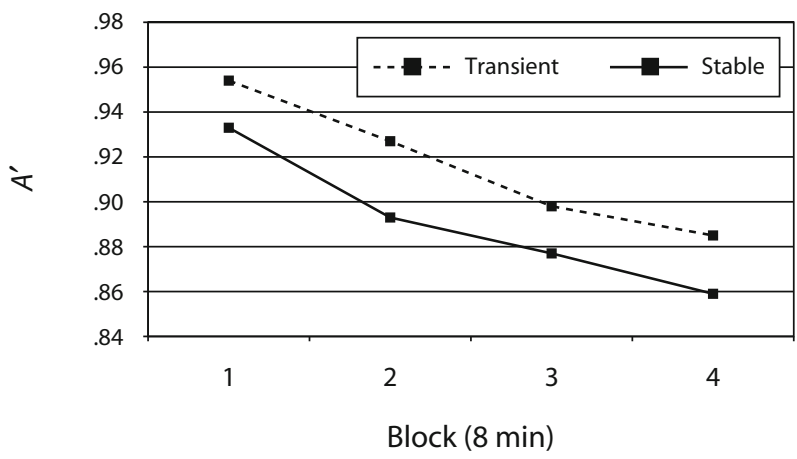

Figure 3. Changes in perceptual sensitivity $\left(A^{\prime}\right)$ as a function of time on task in Experiment 2. $A^{\prime}$ plotted over four contiguous 8-min blocks ( 240 trials each) for each display version (stable and transient).

and thresholds tested after the sustained attention task [before, $M=0.98^{\circ}$; after, $M=0.96^{\circ} ; F(1,38)=0.196$, $p=.66]$. No other sources of variance were significant $(.18<p \mathrm{~s}<.34)$. These results demonstrate that low-level perception, as was measured in a threshold task with similar structure to the actual sustained attention task, did not change after continuous performance.

\section{Performance Over Time}

Perceptual sensitivity $\left(A^{\prime}\right)$ over time was assessed with a mixed-model ANOVA with the between-subjects factor of display and the within-subjects factor of time (Blocks 1-4). A main effect of time demonstrated that perceptual sensitivity declined steadily over time for both display versions [Block 1, $M=.944$; Block 2, $M=$ .910 ; Block $3, M=.887$; Block $4, M=.872 ; F(3,36)=$ $37.7, p<.0001]$. In addition, a main effect of display $[F(1,38)=4.74, p=.036]$ revealed that overall performance was better in the transient display. However, the interaction between display and time was not significant $[F(3,36)=0.547, p=.65]$ (see Figure 3). Thus, the data show that sudden-onset cues improved overall sensitivity but did not prevent a decline in perceptual sensitivity with unpredictable timing.

A similar model of response bias $\left(\beta_{\mathrm{D}}^{\prime \prime}\right)$ revealed a main effect of time [Block 1, $M=.69$; Block 2, $M=.78$; Block 3, $M=.90$; Block $4, M=.83 ; F(3,36)=5.78, p=.002]$, indicating that participants were less likely to respond as time passed. The nonsignificant interaction between display and time $[F(3,36)=1.09, p=.36]$ revealed that this increase in response bias over time was not different between the two displays. Although there was no difference in response bias over time between the two display versions, a significant main effect of display $[F(1,38)=4.11, p=.049]$ indicated that participants exhibited a more liberal overall response bias (i.e., were more likely to respond) in the transient ( $M=$ $.70)$ than in the stable $(M=.90)$ display version.

Finally, trimmed mean correct RTs were submitted to a mixed-model ANOVA with the between-subjects factor of display and the within-subjects factor of time (Blocks 1-4). In line with Experiment 1, RTs significantly increased over time [Block 1, $M=683 \mathrm{msec}$; Block 2,
$M=739$ msec; Block 3, $M=737$ msec; Block 4, $M=$ $780 \mathrm{msec} ; F(3,36)=16.87, p<.0001]$. In addition, a significant interaction between time and display revealed that the pattern of RTs over time differed according to display $[F(3,36)=3.06, p=.04]$. Although participants slowed down over time in both displays, they were able to maintain faster responses to targets for a longer portion of the task in the transient display (see Figure 4). No other sources of variance were statistically significant [main effect of display, $F(1,38)=2.12, p=.15$ ]. These results replicate the pattern of increasing target RTs over time revealed in Experiment 1 but also suggest that RT performance suffered more in the stable version with unpredictable timing.

\section{Effects of Stimulus Timing on Performance Over Time}

To assess the independent effect of stimulus timing on perceptual sensitivity over time, we compared performances between those participants who completed the transient display version with a constant ISI (Experiment $1, N=16$ ) and those who completed the transient display version with a variable ISI (Experiment 2, $N=20$ ). A mixed-model ANOVA with the between-subjects factor of ISI (constant vs. variable) and the within-subjects factor of time (Blocks 1-4) revealed a significant main effect of time $[F(3,32)=13.3, p<.0001]$ that was moderated by

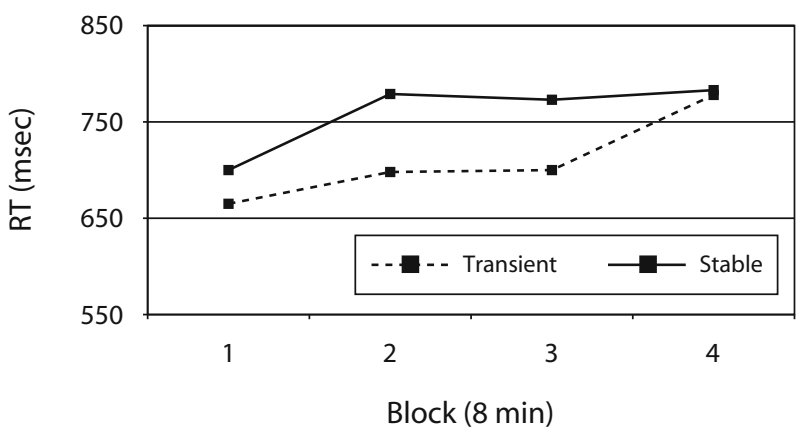

Figure 4. Changes in reaction times (RTs) to targets as a function of time on task in Experiment 2. RTs are plotted over four contiguous 8-min blocks.

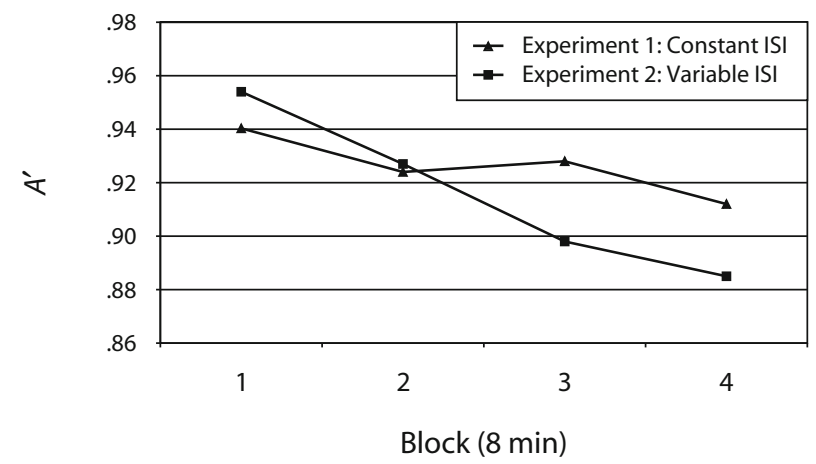

Figure 5. Changes in perceptual sensitivity $\left(A^{\prime}\right)$ as a function of time on task in Experiments 1 and 2 for the transient display version. $A^{\prime}$ is plotted over four contiguous 8 -min blocks ( 240 trials each) for constant (Experiment 1, $N=16$ ) and variable (Experiment 2, $N=20$ ) interstimulus interval (ISI) conditions. 
a significant interaction between time and ISI $[F(3,32)=$ $5.87, p=.003$; see Figure 5]. The main effect of ISI was not significant $(F<1) .{ }^{5}$ The data show that performance over time was better with predictable stimulus timing, controlling for the effect of sudden-onset attention cues.

\section{Discussion}

The results from Experiment 2 revealed that the perceptual benefit due to exogenous cues depended on the endogenous modulation of attention by predictable timing. When stimulus timing was variable, exogenous cues did not prevent a decline in perceptual sensitivity over time. Importantly, exogenous cues did lead to an increase in overall perceptual sensitivity and a decrease in RTs to targets in the transient display. These effects support the conclusion that exogenous cues captured attention and improved performance, even in the face of variable timing, but not to the same extent as when exogenous cues coincided with maximal endogenous attention guided by the implicit endogenous cue of predictable timing, as in Experiment 1. Importantly, predictable timing itself was a strong predictor of successful performance over time, independent of the manipulation of exogenous attention. The results from the comparison of performance over time on the transient tasks in Experiments 1 and 2 demonstrate that, in tasks equated for individual difficulty and initial accuracy, performance declined significantly more over time with variable stimulus timing (Experiment 2).

In addition to changes in perceptual sensitivity over time, we observed an increase in response bias over time in both displays that was not evident in Experiment 1. Increased conservatism over time is a common finding in vigilance studies and is often due to normal changes in probability expectations under conditions of low target probability (Parasuraman \& Davies, 1976). Response bias can also increase as observers come to believe that signal probability is less than they originally thought (See et al., 1997), such as when target probability during a training session influences expectations about target probability in a subsequent sustained attention task (e.g., Colquhoun \& Baddeley, 1964, 1967; Williams, 1986). In Experiment 1, the structure of the threshold procedure was quite different from that of the sustained attention task; thus, participants would have developed no expectation of a particular target probability. In Experiment 2, the structure of the threshold procedure was designed to mimic that of the sustained attention task as much as possible, except with the target occurring more frequently ( $33 \%$ of the time, compared with $10 \%$ of the time in the sustained attention task). Participants likely developed an expectancy regarding target probability while performing the threshold task that later affected their expectancy during the sustained attention task. The observed increase in response bias over time likely reflects the fact that participants became aware that target probability was less than they originally thought, consistent with previous findings.

Although response bias increased over time in both display versions, we observed a more liberal overall response bias in the transient display, which potentially complicates our interpretation of increases in sensitivity. This may have been due to the exogenous cue causing observers to adopt a more liberal decision criterion in terms of responding to targets (see Yeshurun \& Carrasco, 1999, for a discussion). However, the effect of exogenous cues on response bias is usually relevant when cues predict the location of the target (relative to competing locations) or when the cue conveys information about the correct response. In the present study, the exogenous cue did not predict one location over another and did not predict the correct response (i.e., all targets and nontargets occurred in the same location and were all preceded by the cue). It seems more likely that the change in response bias was linked to the change in perceptual sensitivity. In effect, the exogenous cue led to more accurate perception over the course of the task and, thus, to a greater confidence in responding. In signal detection theory, although sensitivity and bias are described theoretically as independent constructs, they are not always found to be independent in practice (Macmillan \& Creelman, 2005, pp. 41-42). In our sample, we found that sensitivity and bias were significantly correlated $[r(39)=-.46, p=.003]$, indicating that higher levels of sensitivity were related to more liberal response bias (lower criterion); we confirmed this significant negative correlation in the sample from Experiment 1 $[r(15)=-.59, p=.016]$. We interpret this relationship in the following way: In a task that presented individually calibrated, purposely ambiguous targets, enhanced perception through a manipulation of exogenous attention seems to have resulted in increased response confidence and a more liberal response bias across individuals.

How does this relationship between sensitivity and bias affect our conclusion regarding shifts in sensitivity with exogenous cues? In cases where sensitivity and bias are estimated from single points on the receiver operating characteristic curve, as was the case here, Macmillan and Creelman (2005, pp. 74-76) described conditions under which sensitivity interpretations remain valid. First, two points with the same bias can always be compared directly (as in Experiment 1). Second, in the case of unequal bias, if the false alarm rate is equal between two conditions, and the hit rate is higher in one condition, "there is no question which represents greater sensitivity" (p. 76). In Experiment 2 , false alarm rates were extremely low in both displays $(<3 \%)$ and, importantly, were not significantly different between displays $[F(1,38)=1.83, p=.18]$. At the same time, overall hit rate was significantly higher in the transient display [transient, $M=.71$; stable, $M=.60$; $F(1,38)=5.90, p=.02]$, in line with the observed significant main effect of display on perceptual sensitivity. Thus, we conclude that the observed increase in overall sensitivity with sudden-onset cues is a reliable indicator of true changes in sensitivity and not a by-product of changes in response bias.

\section{EXPERIMENT 3}

The key finding in Experiment 2 was a significant increase in overall perceptual sensitivity in the transient display that was independent of constant stimulus timing, a finding that we attribute to exogenous attentional capture 
by the sudden-onset cues. However, results from recent studies have suggested that sudden onsets that occur within $100 \mathrm{msec}$ before a subsequent target (and in the same location) can enhance target performance through sensory activation or summation, rather than through exogenous attentional capture (see Richard, Wright, \& Ward, 2003; Wright \& Richard, 2003). In the case of a single precue that occurs on its own, as in Experiments 1 and 2 of the present study, Wright and Richard showed that the total impact of the precue on subsequent target performance is best explained as a combination of sensory activation and exogenous attentional capture. According to the activity distribution model (LaBerge, 1998), the sensory activation contribution is a consequence of a transient increase in activation at the location of the sudden-onset cue that is directly related to its intensity. The aim of Experiment 3 was to test this critical prediction of the sensory activation account - that increased sensory activation from the sudden-onset cue would result in a stronger facilitatory effect. We manipulated the brightness of the sudden-onset cues on a trial-by-trial basis and examined the change in performance over time. If a sensory activation account explains the findings from Experiments 1 and 2, we would expect better performance (higher sensitivity and/ or shorter RTs) on targets following bright cues due to enhanced sensory activation. Alternatively, if an exogenous attention account explains the findings, as we claim, we would expect no difference in performance with changes in cue brightness.

\section{Method}

\section{Participants}

Thirty-seven volunteers (mean age $=20$ years, range $=18-24$ years, 26 female) from the University of California, Davis, gave informed consent and participated in exchange for course credit. Three participants were unable to complete the sustained attention task and were excluded from all analyses. Data from the remaining 34 participants are presented here.

\section{Stimuli}

The transient-display sustained attention task from Experiment 2 was modified to manipulate cue brightness on a trial-by-trial basis. On $50 \%$ of the trials, the mask that occurred before the line was rendered brighter (Michelson contrast ratio between mask and background $=99 \%$ ) than the mask used in Experiments 1 and 2 (Michelson contrast ratio $=97 \%$ ). An equal number of bright and dim cues occurred randomly every 20 trials (i.e., 1 bright target trial and 9 bright nontarget trials were presented every 20 trials). The line and the posttarget mask were equal in luminance to the dim cue (Michelson contrast ratio between bright cue and dim line $=$ $73 \%$ ) on every trial, as in the previous experiments. Participants were informed that the bright cue would occur randomly and that its presentation was unrelated to the occurrence of a target. As in Experiments 1 and 2, the instructions emphasized accuracy in making simple responses with the left mouse button (right index finger) to the short target lines.

\section{Threshold Procedure}

The length of the short target line was set to each participant's $80 \%$ discrimination threshold using the PEST technique, as in Experiment 2. All cues were dim during the threshold procedure, as in Experiment 2. Following the threshold procedure, participants received 5 min of practice on the sustained attention task with intermixed dim and bright cues to ensure that they were able to correctly detect threshold-based targets following cues of differing brightness. Finally, to confirm that the bright cue did not alter baseline perceptual thresholds, participants repeated the threshold procedure with $100 \%$ bright cues at the culmination of the sustained attention task.

\section{Results}

\section{Threshold}

The PEST threshold procedure successfully produced a $\sim .80$ hit rate in all participants ${ }^{6}$ during the first $8 \mathrm{~min}$ of the sustained attention task $(M=.77, S D=.11)$. Importantly, initial hit rates were not significantly different between $\operatorname{dim}(M=.77, S D=.15)$ and bright $(M=.78$, $S D=.15$ ) target trials (paired $t$ test, $t<1$ ), confirming that initial performances were not affected by the brightness of the cue. In addition, there were no significant differences between $\operatorname{dim}\left(M=0.97^{\circ}\right)$ and bright $(M=$ $\left.0.95^{\circ}\right)$ difference thresholds $[t(33)=0.98, p=.33$ ], arguing against baseline perceptual differences between dim and bright cues.

\section{Performance Over Time}

All dependent measures of performance over time were assessed over four continuous blocks of trials (24 targets per block, of which 12 were preceded by a bright cue). Perceptual sensitivity $\left(A^{\prime}\right)$ over time was assessed in a repeated measures ANOVA with the within-subjects factors of time (Blocks 1-4) and cue brightness (bright vs. dim). A main effect of time demonstrated that perceptual sensitivity declined steadily over time [Block 1, $M=$ .933; Block 2, $M=.902$; Block 3, $M=.887$; Block 4, $M=.865 ; F(3,31)=8.55, p<.0001]$, replicating the results of Experiments 1 and 2 . The main effect of cue brightness was not significant $[F(1,33)=1.18, p=.28]$, demonstrating that overall accuracy was not better on bright $(M=.893)$ than on $\operatorname{dim}(M=.901)$ trials, contrary to the prediction of the sensory activation account. The interaction between cue brightness and time was also nonsignificant $[F(3,31)=1.36, p=.27]$, indicating a similar decline in sensitivity over time for bright (Block 1, $M=$ .934; Block 2, $M=.890$; Block 3, $M=.880$; Block 4, $M=.867)$ and $\operatorname{dim}$ (Block $1, M=.933$; Block 2, $M=$ .914; Block 3, $M=.895$; Block 4, $M=.864$ ) trials. In a similar model of response bias, we found a significant increase in response bias over time [Block $1, M=.53$; Block 2, $M=.79$; Block 3, $M=.80$; Block 4, $M=.82$; $F(3,31)=7.09, p=.001]$, as in Experiment 2, but there were no significant differences between dim and bright cues in either overall response bias $[F(1,33)=2.46, p=$ $.13]$ or response bias over time $[F(3,31)=0.11, p=.95]$. Finally, we examined the effects of time (Blocks 1-4) and cue brightness on trimmed mean correct target RTs over time $(N=31$ participants had complete cells for this analysis). In line with Experiments 1 and 2, target RTs significantly increased over time [Block 1, $M=673 \mathrm{msec}$; Block 2, $M=738$ msec; Block 3, $M=753$ msec; Block 4, $M=776$ msec; $F(3,28)=10.23, p<.0001]$. In addition, a main effect of cue brightness revealed that RTs on bright trials were $21 \mathrm{msec}$ longer than those on dim trials [bright, $M=745 \mathrm{msec}$; $\operatorname{dim}, M=724 \mathrm{msec} ; F(1,30)=$ $5.49, p=.026]$. The interaction between time and cue 
brightness was not significant $[F(3,28)=0.24, p=.87]$. These results replicate the pattern of increasing target RTs over time revealed in Experiments 1 and 2 and further demonstrate that the bright mask led to slowing of correct responses, in direct contradiction to the result expected under a sensory activation explanation.

\section{Discussion}

The results from Experiment 3 confirm that the luminance of the sudden-onset cues did not modulate perceptual sensitivity over time: Participants were not more accurate in responding to targets following bright (relative to dim) precues. These results support our conclusion that sudden onsets enhanced perceptual sensitivity in Experiment 2 through exogenous attentional capture, not through sensory summation. In addition, the results from Experiment 3 replicate the finding of decrements in sensitivity and increases in RTs over time with threshold-based targets observed in Experiments 1 and 2 and the finding of increases in response bias observed in Experiment 2. Experiment 3 also confirmed the effectiveness of the PEST procedure in determining threshold-based targets. Finally, the RT data were in line with the perceptual sensitivity findings in that neither supported a sensory activation account, which would predict shorter RTs following bright cues. RTs were, in fact, longer following bright trials. However, it is worth noting that the mean difference in RTs between bright and dim trials was only $21 \mathrm{msec}$; this value represents an extremely small effect size ( $3 \%$ longer than that for dim trials) given the generally long RTs (grand mean $=735 \mathrm{msec}$ ). Thus, the RT data certainly are counter to a sensory activation account; however, we make no claims as to the nature of slight increases in RTs following bright cues.

\section{GENERAL DISCUSSION}

We examined the effect of exogenous and endogenous attention cues on voluntary, sustained visual attention. Using threshold-based targets, we found that perceptual sensitivity declined predictably over time, which was mitigated by the influence of exogenous attention. Importantly, the influence of exogenous attention depended on the endogenous demands of the task. When endogenous attention could be deployed in a systematic fashion using predictable timing information, exogenous cues helped improve performance over time. When stimulus timing was unpredictable, however, exogenous cues did not improve sustained performance. In general, successful performance depended on both efficient deployment of endogenous attention over time due to predictable timing information (Experiment 1) and exogenous sudden-onset cues that attracted attention to the task to increase overall sensitivity (Experiments 1 and 2). Increasing the luminance of the cue did not further improve perceptual sensitivity (Experiment 3 ), supporting the view that the changes that we observed were due to the modulation of attention through exogenous cues, not the result of sensory summation.

These findings support the idea that successful vigilance performance is a result of a dynamic interaction between endogenous attentional control and exogenous events that capture attention, in line with theories that seek to describe the neurobiological basis of attention (Corbetta et al., 2008; Corbetta \& Shulman, 2002). We interpret the effects of exogenous and endogenous attention in light of evidence supporting a resource model of vigilance. Specifically, exogenous cues independently improved overall sensitivity by transiently increasing attentional resources but were arguably not effective in preventing a decline in vigilance. On the other hand, implicit endogenous cues independently improved sustained performance, in line with previous findings (Scerbo et al., 1987). If the major limiting factor during vigilance is the resource demand imposed on the endogenous attention system, it appears that manipulations of endogenous attention that result in the conservation of resources over time are more effective at preventing the vigilance decrement than are exogenous cues that only transiently prime resources. The combined benefit of exogenous and endogenous cues was clearly evident, in that the best performance over time was achieved when exogenous cuing coincided with maximal endogenous attention (Experiment 1, transient display). Taken together, the present data extend previous findings that have posited that errors in sustained attention tasks are a consequence of the limits of effortful, voluntary attention, given high resource demands (Grier et al., 2003; Helton et al., 2005; Helton \& Warm, 2008; Smit et al., 2004; Szalma et al., 2004).

\section{Threshold-Based Targets and Overall Task Demand}

In our tasks, we employed parameters previously shown to increase resource demand and promote declines in perceptual sensitivity, including a high event rate and successive presentation. In addition, we experimentally manipulated resource demands in three different ways: (1) by individualizing target discrimination difficulty using thresholdbased targets; (2) by manipulating display features to vary the degree of exogenous attention drawn to the task; and (3) by manipulating the predictability of stimulus timing, which provided information about when to allocate endogenous attention. We found that a challenging perceptual discrimination placed high demands on the endogenous attention system to maintain performance, in that sensitivity declined reliably over time. In addition, the obtained pattern of slowing of responses to targets over time provides further evidence of a decrement in performance efficiency due to decreased perceptual sensitivity (Davies \& Tune, 1969; Parasuraman, 1986). Given that results in vigilance studies have been shown to be specific to certain task factors that increase resource demands (e.g., high event rate and successive presentation), future research could focus on how exogenous and endogenous cues affect sustained performance when event rate is slow or when a simultaneous presentation design is implemented.

When studying changes in sensitivity over long periods of time, controlling for individual differences in low-level perception is critical. The PEST procedure implemented in Experiments 2 and 3 proved to be an efficient and accurate way to set target parameters, thus creating sustained attention tasks that were equally attentionally and per- 
ceptually challenging to all individuals. Since the PEST method can flexibly converge on any chosen threshold level (within an appropriate range), this approach allows an assessment of sustained attention at varying levels of baseline performance. In effect, standardizing baseline sensitivity helps control the total demand of the task or the amount of effort required for task completion. In a meta-analysis of the sensitivity decrement in 42 vigilance studies, See et al. (1995) suggested that total task demand helps explain the variation in the vigilance decrement not directly attributable to other critical design factors. Our threshold-calibration approach is a promising method for equating overall task difficulty according to individual perceptual ability and, thus, equating the extent of the vigilance decrement across individuals.

\section{Benefits of Sudden Onsets}

We found that resource demands could be reduced by enhancing perception through simple sudden-onset cues. We interpret the improvement in perceptual sensitivity observed in both Experiments 1 and 2 as the beneficial consequence of sudden-onset cues exogenously attracting attentional resources to the display (see the following discussion of the locus of perceptual benefits). Importantly, in Experiment 3, we demonstrated that this improvement could not be explained by a sensory activation account (Wright \& Richard, 2003), in that brighter cues did not improve accuracy. Furthermore, another unique feature of the transient display - the sudden offset of the mask - could not have driven perceptual sensitivity enhancements, since previous studies have shown that sudden changes in luminance that occur after the target stimulus do not enhance performance (Liu, Pestilli, \& Carrasco, 2005). Thus, the improvement in overall sensitivity that occurred with sudden onsets in Experiment 2 supports the independent role of exogenous attention in the perceptual domain. In addition, we observed concomitant changes in target response speed over time. Since accuracy was strongly emphasized over speed in both experiments, we interpret the modulation of target responses as further support that sudden onsets made the perceptual decision easier (Parasuraman, 1986, p. 14). In line with this interpretation, Parasuraman and Davies (1976) examined changes in response latencies in a vigilance task that emphasized accuracy over speed in making different button responses to both targets and nontargets. They found that, although correct detection and false alarm RTs (i.e., positive response latency) increased over time, RTs associated with correct rejections and errors to nontargets (i.e., negative response latency) decreased or remained stable over time. The increase in RTs associated with positive responses was taken as a marker of the relative strength of evidence supporting a positive (target) rather than a negative (nontarget) decision. That is, since observers attempt to be as accurate as possible, correct discrimination of targets is faster in the case where better perception supports the target-nontarget decision. In the present study, sudden onsets led to both better perception and faster responses to targets over time on task.

Importantly, sudden onsets occurred on every trial, and they presumably increased sensitivity on both target and nontarget trials. In a sustained attention task with rare targets, maintaining a reliable percept of the nontarget stimulus is important for accurate identification of the target when it does occur. Indeed, when the percept of the nontarget does not have to be held in working memory, such as in simultaneous-presentation tasks (e.g., deciding whether two lines presented simultaneously are different in length), sensitivity does not reliably decline over time (Parasuraman, 1979). Furthermore, Caggiano and Parasuraman (2004) demonstrated that simultaneous performance of a spatial working memory task interfered with the discrimination of a rare target defined by its spatial location (i.e., eccentricity). They interpreted the increased perceptual sensitivity decrement under the condition of a competing working memory task to reflect the consequence of worse working memory representations of both the standard nontarget position and the infrequent target position. In line with the critical role of memory load in vigilance (Parasuraman, 1979; Parasuraman \& Davies, 1977), the improvement in perception with exogenous attention in the present study could help maintain an accurate working memory representation of the nontarget line, thus making target discrimination easier.

\section{Benefits of Predictable Timing}

Resource demands were also reduced by providing observers with predictable timing information about when to pay maximal attention. Although performance benefits were greatest when sudden onsets were combined with a constant ISI, predictable timing independently improved sustained performance, a finding that confirms the results of previous reports (Scerbo et al., 1987). Studies of attention in both the visual (Martin et al., 2005; Olson \& Chun, 2001) and the auditory (Jones, Moynihan, MacKenzie, \& Puente, 2002) domains have shown that endogenous attention is modulated according to the underlying temporal structure of stimulus events, leading to more accurate performance with predictable timing. This kind of anticipatory attending to specific moments in time has been described as an important mechanism for synchronizing attentional resources with critical external events in a way that can flexibly improve behavior (Large \& Jones, 1999). Moreover, the modulation of endogenous attention in this manner is an efficient way to conserve attentional resources so that maximal attention coincides primarily with taskrelevant events. The present replication of the effects of implicit timing cues on sustained performance points to a critical role for this kind of attentional cuing in preserving limited resources over long periods of time on task.

Temporal predictability can certainly modulate endogenous attention without explicit intention on the part of the observer to voluntarily direct attention differently across time (Martin et al., 2005). Still, it is unclear whether the regular underlying temporal structure in the present study also manipulated voluntary attention explicitly. Previous studies of the effects of both spatial and temporal probability on attention have shown large variability in how much observers notice and volitionally use the underlying expectancies to direct attention during the task (Geng \& Behrmann, 2005). The constant ISI in Experiment 1 
$(1.85 \mathrm{sec})$ provided observers with a relatively long and predictable period during which attention was not required. Since voluntary attention can be generated toward a particular location in space in $\sim 300 \mathrm{msec}$ after the onset of an instructional cue (Müller \& Rabbitt, 1989; Posner \& Cohen, 1984), observers could afford to take a break in attention after presentation of the previous line and then explicitly redirect attention toward the end of the ISI to prepare for the next line. Indeed, studies of temporal attention within the range of the ISI used in our experiments have shown that observers can accurately direct voluntary selective attention to specific moments in time on the basis of instructional cues (Coull, 2004; Coull et al., 2000; Coull \& Nobre, 1998). In terms of temporal complexity, the present study was relatively simple and straightforward, and some participants likely became aware of the regular temporal pattern and used it to take voluntary timeouts during the ISI, then oriented maximal attention to the point in time of the next stimulus. Thus, although we can confidently conclude that temporal regularity modulated endogenous attention implicitly, it is possible that temporal regularity also modulated endogenous attention explicitly.

Given that observers could afford to take an attention timeout, why would they? Although the true upper limit of voluntary attention is unknown, and voluntary attention can be maintained in a given location for many seconds (e.g., Ling \& Carrasco, 2006; Silver, Ress, \& Heeger, 2007), there is evidence that maintaining attention continuously even for relatively short periods of time ( $\sim 5 \mathrm{~min})$ is not always successful, resulting in attentional lapses along with behavioral and neural processing consequences (Weissman et al., 2006). In this vein, there is mounting evidence for a phenomenological basis of mind wandering, or breaks in attention that occur spontaneously during performance of a central task. Behavioral studies using self-reports, thought probes and RT measures (for a review, see Smallwood \& Schooler, 2006), and neuroimaging studies of non-task-related brain activity (Mason et al., 2007; Smallwood, Beach, Schooler, \& Handy, 2008) have demonstrated that observers often experience disruptions in focused attention while performing sustained attention tasks, such as visual monitoring or reading. In this way, voluntary attention is not usually maintained at a maximum level but rather appears to wax and wane over long periods of time. Predictable timing information about when to pay attention can be used to efficiently deploy maximal attention only during presentation of a potential target and, thus, to avoid the consequences of unintentional mind wandering. Future research should continue to investigate the relationship between sustained attentional effort and mind wandering and should examine how implicit and explicit manipulations of endogenous attention can mitigate normal failures of attentional focus.

\section{Combined Benefit of Exogenous \\ Cuing and Predictable Timing}

We observed the best performances over time when exogenous cuing coincided with maximal endogenous attention guided by predictable timing (Experiment 1, transient display). This result is consistent with the general finding that the impact of an exogenous cue on attentional capture depends on endogenous expectations (Folk et al., 1992). Recent evidence demonstrates that temporal expectation, in particular, can strongly influence the impact of an exogenous cue. Milliken et al. (2003) investigated this relationship by manipulating temporal expectancies across two versions of a visual discrimination task: A target could occur more frequently either 1,100 or $1,900 \mathrm{msec}$ after trial onset. On all exogenously cued trials in both task versions, an abrupt luminance change occurred only at the shorter time interval $(1,000 \mathrm{msec}$ after trial onset $)$. Consistent with the classic exogenous cuing effect, Milliken et al. showed that RTs were shorter on cued than on uncued trials when the target occurred $100 \mathrm{msec}$ after the cue. Importantly, this exogenous cuing effect was stronger when participants expected the target to occur at about the time of the cue than when participants expected the target to occur at a later time. Thus, performance benefits were maximal when the exogenous cue coincided with contextual endogenous expectancies about when the target was likely to appear. This finding supports our present result that the full impact of a sudden-onset cue depended on constant and predictable stimulus timing.

\section{The Nature of Perceptual Benefits Due to Exogenous Cuing}

We interpret the improvement in perceptual sensitivity due to sudden onsets in the transient display to be specific to both the spatial location and the timing of the exogenous cue: The sudden-onset cue captured attention exogenously and thus improved perception of the line stimulus that occurred $100 \mathrm{msec}$ later in the same location. In other words, the sudden-onset cue would not have improved perception if it had occurred in another location on the screen or had not occurred within $\sim 200 \mathrm{msec}$ before the line stimulus (for review of the time-limited nature of exogenous cues, see Klein, 2000). This interpretation is in line with many behavioral, electrophysiological, and neuroimaging studies that have described the spatially specific and time-limited perceptual benefits of exogenous attention cues (Carrasco et al., 2002; Jonides \& Yantis, 1988; Ling \& Carrasco, 2006; Liu et al., 2005; Müller \& Rabbitt, 1989; Posner \& Cohen, 1984; Yeshurun \& Carrasco, 1999), including benefits linked to exogenous cues presented within the location of voluntary attention (Hopfinger \& West, 2006) and at the fovea (Coull et al., 2000). In support of the strong effects of sudden onsets on attentional capture and subsequent improvements in perception, Hopfinger and Maxwell (2005) elegantly demonstrated that sudden changes in luminance triggered enhancements in early visual cortical responses (P1 ERP amplitude) to subsequent visual stimuli that occurred at the cued location, even when the cues were not predictive of the upcoming stimuli, the stimuli themselves were entirely task irrelevant (i.e., intentionally ignored), and the stimuli did not require decisions or responses. This finding supports our interpretation that the sudden-onset cues used in the present study reflexively captured attention and enhanced processing of the subsequent line stimuli. In addition, the full impact of exogenous cues has been shown to depend on how much the cue shares in common 
with task-relevant target features (Folk et al., 2002). In the present design, cue and line stimuli were the same color and luminance (cf. Experiment 3 ) and overlapped spatially, lending support to the conclusion that the sudden-onset cues that we used maximally captured exogenous attention. Thus, several lines of evidence support our conclusion that the perceptual sensitivity enhancements in the transient display were directly driven by the capture of exogenous attention by the sudden-onset cues.

However, it is imperative to also consider an alternative explanation: Performance improvements could have been due to some other aspect of the cue, such as the regularity or pattern of its occurrence (e.g., the cues always appeared at the same location, with the same basic timing, same size, same luminance, etc.). Specifically, given the demonstrated role of regularity of stimulus timing on sustained performance (Experiment 2), it is important to consider whether the exogenous cue could have provided regular timing information. Early studies of exogenous attention demonstrated behavioral enhancements due to spatially specific precues using variable cue-target intervals (Müller \& Rabbitt, 1989). However, many recent studies have used a regular cue-target interval in the range of the most effective timing parameters determined from the early studies, in line with the present approach. Importantly, spatially nonspecific precues (neutral cues) that only provide timing information do not improve perceptual sensitivity. Carrasco et al. (2004) showed that, when both neutral and spatially specific precues occurred $120 \mathrm{msec}$ before the upcoming stimulus, contrast sensitivity only improved with spatially specific precues. Thus, we do not interpret improvements in perceptual sensitivity in the transient display to be due to aspects of timing regularity. Although we did not directly manipulate cue-target timing in the present study, we did explicitly disrupt another aspect of the regular cue-target pattern by changing the luminance of the cue on a trial-by-trial basis in Experiment 3. Our findings show that random, unexpected changes in cue luminance did not alter performance, suggesting that at least the regularity of the brightness of the cue did not contribute strongly to our findings. Overall, the most parsimonious explanation of enhanced performance in the transient display is an increase in perceptual sensitivity following the sudden-onset cue, in line with previous findings of enhanced sensitivity with exogenous attention. Still, future research on the impact of variable cue-target timing and other aspects of cue regularity on exogenous effects would be valuable to a full understanding of both short-duration attention and vigilance.

\section{CONCLUSION}

In conclusion, our results suggest a strong interaction between endogenous and exogenous attention during vigilance performance. The results from Experiment 2 (Figure 3) confirm that sudden onsets increased overall perceptual sensitivity in a manner consistent with changes in perception following exogenous spatial cues. Furthermore, predictable stimulus timing significantly reduced the sensitivity decrement, independent of the effect of sudden onsets (Figure 5). On the basis of this finding, we conclude that endogenous attention was modulated by the predictable nature of the stimuli, such that maximum attentional focus coincided with stimulus presentation. The combined impact of exogenous cues and predictable timing (Experiment 1) reinforces the proposal that sudden onsets are maximally effective when regular patterns of stimulus presentation support optimal, endogenous preparation for the upcoming stimulus (Milliken et al., 2003).

\section{AUTHOR NOTE}

This study was supported by a National Science Foundation Graduate Research Fellowship to K.A.M., by Grants MH057714 and NSF0727115 to G.R.M., and by John E. Fetzer Institute Grant 2191 to C.D.S. We thank Michael X Cohen for programming assistance, and Joy J. Geng, Anthony P. Zanesco, Bong J. Walsh, and anonymous reviewers for helpful suggestions on previous versions of the manuscript. Correspondence concerning this article should be addressed to K. A. MacLean, 267 Cousteau Place, Center for Mind and Brain, Davis, CA 95618 (e-mail: katherine.a.maclean@gmail.com).

\section{REFERENCES}

BAKAN, P. (1955). Discrimination decrement as a function of time in a prolonged vigil. Journal of Experimental Psychology, 50, 387-390. doi:10.1037/h0044041

Berger, C., \& Mahneke, A. (1954). Fatigue in two simple visual tasks. American Journal of Psychology, 67, 509-512. doi:10.2307/1417942

Caggiano, D. M., \& Parasuraman, R. (2004). The role of memory representation in the vigilance decrement. Psychonomic Bulletin \& Review, 11, 932-937.

Carrasco, M., Ling, S., \& Read, S. (2004). Attention alters appearance. Nature Neuroscience, 7, 308-313. doi:10.1038/nn1194

Carrasco, M., Penpeci-Talgar, C., \& Eckstein, M. (2000). Spatial covert attention increases contrast sensitivity across the CSF: Support for signal enhancement. Vision Research, 40, 1203-1215. doi:10.1016/ S0042-6989(00)00024-9

Carrasco, M., Williams, P. E., \& Yeshurun, Y. (2002). Covert attention increases spatial resolution with or without masks: Support for signal enhancement. Journal of Vision, 2, 467-479. doi:10.1167/2.6.4

Chun, M. M., \& Jiang, Y. (1998). Contextual cueing: Implicit learning and memory of visual context guides spatial attention. Cognitive Psychology, 36, 28-71. doi:10.1006/cogp.1998.0681

Colquhoun, W. P., \& BAdDeley, A. D. (1964). Role of pretest expectancy in vigilance decrement. Journal of Experimental Psychology, 68, 156-160. doi:10.1037/h0042875

Colquhoun, W. P., \& Baddeley, A. D. (1967). Influence of signal probability during pretraining on vigilance decrement. Journal of Experimental Psychology, 73, 153-155. doi:10.1037/h0024087

Corbetta, M., Patel, G., \& Shulman, G. L. (2008). The reorienting system of the human brain: From environment to theory of mind. Neuron, 58, 306-324. doi:10.1016/j.neuron.2008.04.017

Corbetta, M., \& Shulman, G. L. (2002). Control of goal-directed and stimulus-driven attention in the brain. Nature Reviews Neuroscience, 3, 201-215. doi: $10.1038 /$ nrn755

Coren, S., WARD, L. M., \& EnNs, J. T. (1999). Sensation and perception (5th ed.). Fort Worth, TX: Harcourt-Brace.

Coull, J. T. (2004). fMRI studies of temporal attention: Allocating attention within, or towards, time. Cognitive Brain Research, 21, 216226. doi:10.1016/j.cogbrainres.2004.02.011

Coull, J. T., Frith, C. D., Büchel, C., \& Nobre, A. C. (2000). Orienting attention in time: Behavioural and neuroanatomical distinction between exogenous and endogenous shifts. Neuropsychologia, 38, 808-819. doi:10.1016/S0028-3932(99)00132-3

Coull, J. T., \& Nobre, A. C. (1998). Where and when to pay attention: The neural systems for directing attention to spatial locations and to time intervals as revealed by both PET and fMRI. Journal of Neuroscience, 18, 7426-7435.

DAVIES, D. R., \& PARASURAMAN, R. (1982). The psychology of vigilance. London: Academic Press. 
Davies, D. R., \& TunE, G. S. (1969). Human vigilance performance. New York: American Elsevier.

Fecteau, J. H., \& Munoz, D. P. (2006). Salience, relevance, and firing: A priority map for target selection. Trends in Cognitive Sciences, 10, 382-390. doi:10.1016/j.tics.2006.06.011

Folk, C. L., Leber, A. B., \& Egeth, H. E. (2002). Made you blink! Contingent attentional capture produces a spatial blink. Perception \& Psychophysics, 64, 741-753.

Folk, C. L., Remington, R. W., \& Johnston, J. C. (1992). Involuntary covert orienting is contingent on attentional control settings. Journal of Experimental Psychology: Human Perception \& Performance, 18, 1030-1044. doi:10.1037/0096-1523.18.4.1030

Frankmann, J. P., \& Adams, J. A. (1962). Theories of vigilance. Psychological Bulletin, 59, 257-272. doi:10.1037/h0046142

Frome, F. S., MacLeod, D. I. A., Buck, S. L., \& Williams, D. R. (1981). Large loss of visual sensitivity to flashed peripheral targets. Vision Research, 21, 1323-1328. doi:10.1016/0042-6989(81)90238-8

Geng, J. J., \& Behrmann, M. (2005). Spatial probability as an attentional cue in visual search. Perception \& Psychophysics, 67, 1252-1268.

Gottlieb, J. (2007). From thought to action: The parietal cortex as a bridge between perception, action, and cognition. Neuron, 53, 9-16. doi:10.1016/j.neuron.2006.12.009

GreEn, D. M., \& Swets, J. A. (1966). Signal detection theory and psychophysics. New York: Wiley.

Grier, R. A., Warm, J. S., Dember, W. N., Matthews, G., Galinsky, T. L., Szalma, J. L., \& Parasuraman, R. (2003). The vigilance decrement reflects limitations in effortful attention, not mindlessness. Human Factors, 45, 349-359. doi:10.1518/hfes.45.3.349.27253

Helton, W. S., Hollander, T. D., Warm, J. S., Matthews, G., DemBER, W. N., WALLAart, M., ET AL. (2005). Signal regularity and the mindlessness model of vigilance. British Journal of Psychology, 96, 249-261. doi:10.1348/000712605X38369

Helton, W. S., \& Warm, J. S. (2008). Signal salience and the mindlessness theory of vigilance. Acta Psychologica, 129, 18-25. doi:10.1016/j .actpsy.2008.04.002

HitchCock, E. M., Dember, W. N., Warm, J. S., Moroney, B. W., \& SEE, J. E. (1999). Effects of cueing and knowledge of results on workload and boredom in sustained attention. Human Factors, 41, 365-372. doi:10.1518/001872099779610987

Hitchсоск, E. M., Warm, J. S., Matthews, G., Dember, W. N., SheAr, P. K., TripP, L. D., ET AL. (2003). Automation cueing modulates cerebral blood flow and vigilance in a simulated air traffic control task. Theoretical Issues in Ergonomics Science, 4, 89-112. doi:10.1080/14639220210159726

Hoffmann, J., \& Kunde, W. (1999). Location-specific target expectancies in visual search. Journal of Experimental Psychology: Human Perception \& Performance, 25, 1127-1141. doi:10.1037/0096 $-1523.25 .4 .1127$

Hopfinger, J. B., \& MAXwell, J. S. (2005). Appearing and disappearing stimuli trigger a reflexive modulation of visual cortical activity. Brain Research: Cognitive Brain Research, 25, 48-56. doi:10.1016/j .cogbrainres.2005.04.010

Hopfinger, J. B., \& West, V. M. (2006). Interactions between endogenous and exogenous attention on cortical visual processing. NeuroImage, 31, 774-789. doi:10.1016/j.neuroimage.2005.12.049

Jones, M. R., Moynihan, H., MacKenzie, N., \& Puente, J. (2002). Temporal aspects of stimulus-driven attending in dynamic arrays. Psychological Science, 13, 313-319. doi:10.1111/1467-9280.00458

Jonides, J., \& YANTIS, S. (1988). Uniqueness of abrupt visual onset in capturing attention. Perception \& Psychophysics, 43, 346-354.

KLeIN, R. M. (2000). Inhibition of return. Trends in Cognitive Sciences, 4, 138-147. doi:10.1016/S1364-6613(00)01452-2

LABERge, D. (1998). Attentional emphasis in visual orienting and resolving. In R. D. Wright (Ed.), Visual attention (pp. 417-454). New York: Oxford University Press.

Large, E. W., \& Jones, M. R. (1999). The dynamics of attending: How people track time-varying events. Psychological Review, 106, 119159. doi:10.1037/0033-295X.106.1.119

LEeK, M. R. (2001). Adaptive procedures in psychophysical research. Perception \& Psychophysics, 63, 1279-1292.

Ling, S., \& Carrasco, M. (2006). When sustained attention impairs perception. Nature Neuroscience, 9, 1243-1245. doi:10.1038/nn1761

liu, T., Pestilli, F., \& Carrasco, M. (2005). Transient attention en- hances perceptual performance and fMRI response in human visual cortex. Neuron, 45, 469-477. doi:10.1016/j.neuron.2004.12.039

MackWorth, N. H. (1948). The breakdown of vigilance during prolonged visual search. Quarterly Journal of Experimental Psychology, 1, 6-21.

Macmillan, N. A., \& Creelman, C. D. (2005). Detection theory: A user's guide (2nd ed.). Mahwah, NJ: Erlbaum.

Mangun, G. R., \& Hillyard, S. A. (1990). Allocation of visual attention to spatial locations: Tradeoff functions for event-related brain potentials and detection performance. Perception \& Psychophysics, 47, 532-550.

Mangun, G. R., \& Hillyard, S. A. (1991). Modulations of sensoryevoked brain potentials indicate changes in perceptual processing during visual-spatial priming. Journal of Experimental Psychology: Human Perception \& Performance, 17, 1057-1074. doi:10.1037/0096 $-1523.17 .4 .1057$

Manly, T., Robertson, I. H., Galloway, M., \& Hawkins, K. (1999). The absent mind: Further investigations of sustained attention to response. Neuropsychologia, 37, 661-670. doi:10.1016/S0028 $-3932(98) 00127-4$

Martin, T., Egly, R., Houck, J. M., Bish, J. P., Barrera, B. D., Lee, D. C., \& Tesche, C. D. (2005). Chronometric evidence for entrained attention. Perception \& Psychophysics, 67, 168-184.

Martínez, A., Di Russo, F., Anllo-Vento, L., \& Hillyard, S. A. (2001). Electrophysiological analysis of cortical mechanisms of selective attention to high and low spatial frequencies. Clinical Neurophysiology, 112, 1980-1998. doi:10.1016/S1388-2457(01)00660-5

Mason, M. F., Norton, M. I., Van Horn, J. D., Wegner, D. M., Grafton, S. T., \& Macrae, C. N. (2007). Wandering minds: The default network and stimulus-independent thought. Science, 315, 393395. doi: $10.1126 /$ science. 1131295

Maxwell, S. E., \& Delaney, H. D. (2004). Designing experiments and analyzing data: A model comparison perspective (2nd ed.). Mahwah, NJ: Erlbaum.

Milliken, B., Lupiáñez, J., Roberts, M., \& Stevanovski, B. (2003). Orienting in space and time: Joint contributions to exogenous spatial cuing effects. Psychonomic Bulletin \& Review, 10, 877-883.

Miniussi, C., RaO, A., \& Nobre, A. C. (2002). Watching where you look: Modulation of visual processing of foveal stimuli by spatial attention. Neuropsychologia, 40, 2448-2460. doi:10.1016/S0028 $-3932(02) 00080-5$

Most, S. B., Simons, D. J., Scholl, B. J., Jimenez, R., Clifford, E., \& Chabris, C. F. (2001). How not to be seen: The contribution of similarity and selective ignoring to sustained inattentional blindness. Psychological Science, 12, 9-17. doi:10.1111/1467-9280.00303

MülLER, H. J., \& RabBITt, P. M. A. (1989). Reflexive and voluntary orienting of visual attention: Time course of activation and resistance to interruption. Journal of Experimental Psychology: Human Perception \& Performance, 15, 315-330. doi:10.1037/0096-1523.15.2.315

Nuechterlein, K. H., Parasuraman, R., \& Jiang, Q. (1983). Visual sustained attention: Image degradation produces rapid sensitivity decrement over time. Science, 220, 327-329. doi:10.1126/ science. 6836276

O’Connell, R. G., Bellgrove, M. A., Dockree, P. M., Lau, A., Fitzgerald, M., \& Robertson, I. H. (2008). Self-Alert Training: Volitional modulation of autonomic arousal improves sustained attention. Neuropsychologia, 46, 1379-1390. doi:10.1016/j .neuropsychologia.2007.12.018

Olson, I. R., \& Chun, M. M. (2001). Temporal contextual cuing of visual attention. Journal of Experimental Psychology: Learning, Memory, \& Cognition, 27, 1299-1313. doi:10.1037/0278-7393.27.5.1299

Parasuraman, R. (1979). Memory load and event rate control sensitivity decrements in sustained attention. Science, 205, 924-927. doi: $10.1126 /$ science. 472714

Parasuraman, R. (1986). Vigilance, monitoring and search. In K. R. Boff, L. Kaufman, \& J. P. Thomas (Eds.), Handbook of perception and human performance: Vol. 2. Cognitive processes and performance (pp. 1-39). New York: Wiley.

Parasuraman, R., \& Davies, D. R. (1976). Decision theory analysis of response latencies in vigilance. Journal of Experimental Psychology: Human Perception \& Performance, 2, 578-590. doi:10.1037/0096 $-1523.2 .4 .578$

Parasuraman, R., \& Davies, D. R. (1977). A taxonomic analysis of 
vigilance. In R. R. Mackie (Ed.), Vigilance: Theory, operational performance, and physiological correlates (pp. 559-574). New York: Plenum.

Parasuraman, R., \& Mouloua, M. (1987). Interaction of signal discriminability and task type in vigilance decrement. Perception \& Psychophysics, 41, 17-22.

Paus, T., Zatorre, R. J., Hofle, N., Caramanos, Z., Gotman, J., Petrides, M., \& Evans, A. C. (1997). Time-related changes in neural systems underlying attention and arousal during the performance of an auditory vigilance task. Journal of Cognitive Neuroscience, 9, 392408. doi:10.1162/jocn.1997.9.3.392

Posner, M. I. (1980). Orienting of attention. Quarterly Journal of Experimental Psychology, 32, 3-25.

Posner, M., \& CoHEn, Y. (1984). Components of attention. In H. Bouma \& D. G. Bouwhuis (Eds.), Attention and performance X: Control of language processes (pp. 531-556). Hillsdale, NJ: Erlbaum.

RichaRD, C. M., Wright, R. D., \& WARD, L. M. (2003). Goal-driven modulation of stimulus-driven attentional capture in multiple-cue displays. Perception \& Psychophysics, 65, 939-955.

Robertson, I. H., Manly, T., Andrade, J., Baddeley, B. T., \& YIEND, J. (1997). “Oops!”: Performance correlates of everyday attentional failures in traumatic brain injured and normal subjects. Neuropsychologia, 35, 747-758. doi:10.1016/S0028-3932(97)00015-8

Robertson, I. H., Mattingley, J. B., Rorden, C., \& Driver, J. (1998). Phasic alerting of neglect patients overcomes their spatial deficit in visual awareness. Nature, 395, 169-172. doi:10.1038/25993

Robertson, I. H., Tegnér, R., Tham, K., Lo, A., \& Nimmo-Smith, I. (1995). Sustained attention training for unilateral neglect: Theoretical and rehabilitation implications. Journal of Clinical \& Experimental Neuropsychology, 17, 416-430. doi:10.1080/01688639508405133

RuZ, M., \& LupiáÑ̃E, J. (2002). A review of attentional capture: On its automaticity and sensitivity to endogenous control. Psicológica, 23, 283-309.

Santangelo, V., \& Spence, C. (2008). Is the exogenous orienting of spatial attention truly automatic? Evidence from unimodal and multisensory studies. Consciousness \& Cognition, 17, 989-1015. doi:10.1016/j.concog.2008.02.006

Scerbo, M. W., Warm, J. S., Doettling, V. S., Parasuraman, R., \& FISK, A. D. (1987). Event asynchrony and task demands in sustained attention. In L. S. Mark, J. S. Warm, \& R. L. Huston (Eds.), Ergonomics and human factors: Recent research (pp. 33-39). New York: Springer.

See, J. E., Howe, S. R., Warm, J. S., \& Dember, W. N. (1995). Metaanalysis of the sensitivity decrement in vigilance. Psychological Bulletin, 117, 230-249. doi:10.1037/0033-2909.117.2.230

See, J. E., Warm, J. S., Dember, W. N., \& Howe, S. R. (1997). Vigilance and signal detection theory: An empirical evaluation of five measures of response bias. Human Factors, 39, 14-29. doi:10.1518/ 001872097778940704

Serences, J. T., Shomstein, S., Leber, A. B., Golay, X., Egeth, H. E., \& YANTIS, S. (2005). Coordination of voluntary and stimulus-driven attentional control in human cortex. Psychological Science, 16, 114122. doi:10.1111/j.0956-7976.2005.00791.x

Silver, M. A., Ress, D., \& HeEger, D. J. (2007). Neural correlates of sustained spatial attention in human early visual cortex. Journal of Neurophysiology, 97, 229-237. doi:10.1152/jn.00677.2006

Simons, D. J., \& Chabris, C. F. (1999). Gorillas in our midst: Sustained inattentional blindness for dynamic events. Perception, 28, 1059-1074. doi: $10.1068 / \mathrm{p} 2952$

Smallwood, J., Beach, E., Schooler, J. W., \& Handy, T. C. (2008). Going AWOL in the brain: Mind wandering reduces cortical analysis of external events. Journal of Cognitive Neuroscience, 20, 458-469. doi:10.1162/jocn.2008.20.3.458

Smallwood, J., \& Schooler, J. W. (2006). The restless mind. Psychological Bulletin, 132, 946-958. doi:10.1037/0033-2909.132.6.946

Smit, A. S., Eling, P. A. T. M., \& Coenen, A. M. L. (2004). Mental effort causes vigilance decrease due to resource depletion. Acta Psychologica, 115, 35-42. doi:10.1016/j.actpsy.2003.11.001

Stanislaw, H., \& Todorov, N. (1999). Calculation of signal detection theory measures. Behavior Research Methods, Instruments, \& Computers, 31, 137-149.

Steinman, B. A., Steinman, S. B., \& Lehmkuhle, S. (1997). Transient visual attention is dominated by the magnocellular stream. Vision Research, 37, 17-23. doi:10.1016/S0042-6989(96)00151-4
Stroop, J. R. (1935). Studies of interference in serial verbal reactions. Journal of Experimental Psychology, 18, 643-662. doi:10.1037/ h0054651

Szalma, J. L., Warm, J. S., Matthews, G., Dember, W. N., Weiler, E. M., Meier, A., \& Eggemeier, F. T. (2004). Effects of sensory modality and task duration on performance, workload, and stress in sustained attention. Human Factors, 46, 219-233. doi:10.1518/ hfes.46.2.219.37334

Taylor, M. M., \& Creelman, C. D. (1967). PEST: Efficient estimates on probability functions. Journal of the Acoustical Society of America, 41, 782-787. doi:10.1121/1.1910407

Theeuwes, J. (1991). Exogenous and endogenous control of attention: The effect of visual onsets and offsets. Perception \& Psychophysics, 49, 83-90.

VAN DER Lubbe, R. H. J., \& Postma, A. (2005). Interruption from irrelevant auditory and visual onsets even when attention is in a focused state. Experimental Brain Research, 164,464-471. doi:10.1007/s00221 $-005-2267-0$

Van Voorhis, S. [T.], \& Hillyard, S. A. (1977). Visual evoked potentials and selective attention to points in space. Perception \& Psychophysics, 22, 54-62.

WARM, J. S., \& JERISON, H. J. (1984). The psychophysics of vigilance. In J. S. Warm (Ed.), Sustained attention in human performance (pp. 1559). Chichester, U.K.: Wiley.

Warm, J. S., Parasuraman, R., \& Matthews, G. (2008). Vigilance requires hard mental work and is stressful. Human Factors, 50, 433441. doi: $10.1518 / 001872008$ X312152

Weissman, D. H., Roberts, K. C., Visscher, K. M. \& WoldorfF, M. G. (2006). The neural bases of momentary lapses in attention. $\mathrm{Na}$ ture Neuroscience, 9, 971-978. doi:10.1038/nn1727

WIENER, E. L. (1973). Adaptive measurement of vigilance decrement. Ergonomics, 16, 353-363. doi:10.1080/00140137308924527

Williams, P. S. (1986). Processing demands, training, and the vigilance decrement. Human Factors, 28, 567-579.

Wright, R. D., \& Richard, C. M. (2003). Sensory mediation of stimulus-driven attentional capture in multiple-cue displays. Perception \& Psychophysics, 65, 925-938.

YANTIS, S., \& Jonides, J. (1990). Abrupt visual onsets and selective attention: Voluntary versus automatic allocation. Journal of Experimental Psychology: Human Perception \& Performance, 16, 121-134. doi:10.1037/0096-1523.16.1.121

Yeshurun, Y., \& CARRASCO, M. (1999). Spatial attention improves performance in spatial resolution tasks. Vision Research, 39, 293-306. doi:10.1016/S0042-6989(98)00114-X

\section{NOTES}

1. One participant received extra practice and subsequently performed at $87 \%$ accuracy during the first block of the task, well within the range of normal group performance ( $<1 S D$ from the grand mean).

2 . There were 2 participants who were outliers during the first block of the stable display ( $>2 S D$ from the grand mean). These participants performed within $1 S D$ of the grand mean during the first block of the transient display and their thresholds were within $1 S D$ of the grand mean for both displays.

3. Including order as a factor in analyses of RTs did not change the reported results.

4 . There were 2 participants who were outliers during the first block of the stable display ( $>2 S D$ from the grand mean). Their thresholds were within $1 S D$ of the grand mean, and their performances declined steadily over time as in the group data.

5. In an alternate analysis, we randomly selected 16 participants from the sample of 20 participants in Experiment 2 and compared their performances over time with that of the 16 participants in Experiment 1. The main effect of time was again significant $[F(3,28)=12.8, p<.0001]$, as was the interaction between time and ISI $[F(3,28)=5.11, p=.006]$. The main effect of ISI was not significant $(F<1)$.

6 . No participant's performance was $>2 S D$ from the grand mean.

(Manuscript received August 4, 2008; revision accepted for publication January 31, 2009.) 